\title{
Restrukturierung nach der InsO: Gesetzesplan, Fehlstellen und Reformansätze innerhalb einer umfassenden InsO-Novellierung aus Sicht eines Insolvenzpraktikers
}

\author{
Dr. iur. Michael JafFé, München*
}

Inhaltsübersicht

ZGR 2010, 248-263

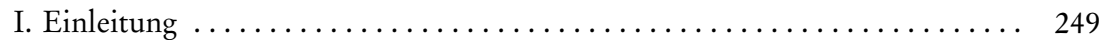

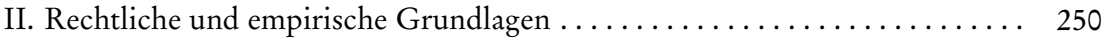

III. USA als Vorbild ? . . . . . . . . . . . . . . . . . . . . 253

IV. Ist die Überschuldung als Insolvenzgrund noch zeitgemäß? . . . . . . . 255

V. Verfahrenskontrolle als Sanierungshebel ?................ 256

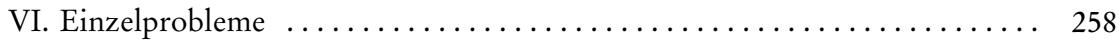

VII. Vorschlagsrecht zur Sanierung $\ldots \ldots \ldots \ldots \ldots \ldots \ldots \ldots \ldots \ldots \ldots \ldots . \ldots \ldots$

VIII. Stellung des Insolvenzgerichts im modernen Insolvenzgeschehen . . . . . 261

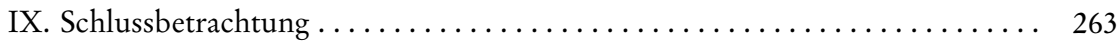

Nach einer kurzen einleitenden Darstellung der auf den Gesetzgeber zukommenden Aufgaben stellt der Verfasser zunächst die rechtlichen sowie empirischen Grundlagen für mögliche Reformansätze dar. Während seiner Ausfübrungen nimmt der Verfasser zudem zu den in dem Beitrag von Herrn Prof. Hirte aufgestellten Thesen an verschiedenen Stellen kritisch Stellung. Sodann setzt er sich mit der Frage auseinander, ob Regelungen des Insolvenzrechts $\operatorname{der}$ USA als Vorbild für deutsche Reformansätze heranzuziehen sein können und kommt zu dem Ergebnis, dass das anglo-amerikanische Rechtssystem durchaus in zentralen Aspekten wie der Konzentration und Spezialisierung der Insolvenzgerichte sowie der Exklusivität für das Vorschlagsrecht bei der Erstellung eines Sanierungsplans überzengen kann. Ebenso wird in diesem Kontext diskutiert, inwieweit die Kontrolle des Verfabrens als Sanierungshebel angesehen werden kann. Im Weiteren folgen Ausfübrungen zu der Frage, inwieweit der Insolvenzgrund der Überschuldung noch zeitgemäß ist und welche Folgen die oft geforderte Streichung nach sich ziehen würde. Der Verfasser fordert die Möglichkeit eines „Debt-toequity-swaps" im Insolvenzplanverfahren zuzulassen, sowie die Rechtsmittellastigkeit durch die Einführung eines Spruchverfahrens zu entschärfen. Zum Abschluss folgt eine konstruktive Betrachtung der Stellung des Insolvenzgerichts im modernen Insolvenzgeschehen unter verständiger Würdigung der Handhabung in den USA.

Following a brief introductory description of the tasks which legislation will be required to address, the author first explains the legal and empirical bases for possible approaches to reform. At various points in the course of his discourse, the author also comments critically on

* Fachanwalt für Steuerrecht, Fachanwalt für Insolvenzrecht, Insolvenzverwalter, Lehrbeauftragter der Ludwig-Maximilians-Universität, München. 
the theories propounded by Prof. Hirte. He then addresses the issue of whether US insolvency law should be used as a role model for German reform initiatives and comes to the conclusion that the Anglo-American legal system may well be convincing in central aspects, such as the concentration and specialisation of the insolvency courts, as well as the exclusivity for the right of proposal in the production of a reorganisation plan. Likewise, the extent to which control of the procedure can be regarded as a lever on reorganisation is also discussed in this context. This is followed by a discussion of the extent to which over-indebtedness as a reason for insolvency is still up-to-date and the consequences which would result from its frequently demanded abolition. The author argues in favour of permitting the introduction of a "debt-to-equityswap" within the framework of the insolvency plan procedure, as well as relief from the burden of appeals by the introduction of award proceedings. The author concludes with a constructive assessment of the position of the insolvency court in modern insolvency with a knowledgeable appreciation of practice in the USA.

\section{Einleitung}

Da das Thema Insolvenz aufgrund der lange noch nicht ausgestandenen Finanz- und Wirtschaftskrise sowie spektakulärer Krisenfälle und Großinsolvenzen im Jahre $2009^{1}$ - auch in der medialen Wahrnehmung - eine dominante Stellung in der öffentlichen Diskussion einnahm und in 2010 weiter einnimmt, verwundert es nicht, dass ein legislativer Änderungsbedarf der Insolvenzordnung auf der politischen Agenda weit oben steht. ${ }^{2} \mathrm{Z}$ war ist dies nicht neu, $\mathrm{da}$ bereits in der letzten Legislaturperiode mehrere Arbeitsgruppen am BMJ eingesetzt waren, um eine Weiterentwicklung der Insolvenzordnung zu forcieren. Es verdichtet sich aber der Eindruck, dass der Gesetzgeber zeitnah agieren wird, um seine Handlungsfähigkeit unter Beweis zu stellen. ${ }^{3}$ Dass dies - trotz Diskontinuität von Gesetzesvorhaben nach Ablauf einer Legislaturperiode - fachlich überhaupt möglich ist, ist dem Umstand zuzuschreiben, dass die erfahrenen Kompetenzträger im BMJ im Fachbereich Rechtspflege (zu dem das Insolvenzrecht gehört) durch die neue Regierung erfreulicherweise beibehalten wurden. Dies gibt die Gewähr, dass einerseits mutige Veränderungen möglich sind, andererseits ein seit 1877 fortentwickeltes und auf römischen Rechtswurzeln fußendes ausgewogenes Konzept zum Umgang mit Unternehmenszusammenbrüchen nicht mit unabsehbaren Folgen leichtfertig über Bord geworfen werden wird.

1 Großinsolvenzen des Handels- und Touristikkonzerns Arcandor sowie dessen Versandhandelssparte mit dem Hauptgeschäft Quelle, des Chiphersteller Qimonda, des Modekonzerns Escada, sowie von Schiesser, Rosenthal, Märklin, des Handelsdiscounters Woolworth, sowie eine Reihe von Firmenzusammenbrüchen in der Automobilindustrie, insbesondere Karmann, Edscha und Askys.

2 Koalitionsvertrag CDU/CSU-FDP, 17. Legislaturperiode, S. 18; Zypries, Symposium zum Konzerninsolvenzrecht, 22. November 2007.

3 Koalitionsvertrag CDU/CSU-FDP, 17. Legislaturperiode, S. 18. 
Die Kunst des Gesetzgebers wird dabei darin liegen, aus den vielfältigen und oft mit guten Gründen gegensätzlichen Argumenten, ein stimmiges Gesamtkonzept zu modellieren, in dem neben rechtlichen Aspekten auch föderale Begrenztheiten eine zentrale Rolle spielen werden. Unstreitig bedarf es legislativer Änderungen, um den aktuellen Anforderungen gerecht zu werden. Die Problemlagen haben sich seit Einführung der Insolvenzordnung am 1. Januar 1999 dramatisch verändert. Zeiten, in denen Unternehmen oder ganze Industriezweige, die über Jahrzehnte die verlässlichen Stützen unseres Gemeinwohls, des volkswirtschaftlichen Wohlstandes und des sozialen Friedens waren, über Nacht Umsatzeinbrüche von bis zu 50 \% überleben müssen ${ }^{4}$, sind in der Nachkriegsgeschichte neu. Demgemäß kann Insolvenz heute nicht mehr simplifizierend mit selbstverschuldetem unternehmerischem Fehlverhalten gleichgesetzt werden, welches - nicht selten mit einer gewissen Häme - stets und gerne auch öffentlich unterstellt wird.

Wenngleich es gesellschaftspolitisch aktuell schwierig ist, der Insolvenz ein positives Momentum abzugewinnen, so muss doch versucht werden, die Berührungsängste mit dem „institutionalisierten Scheitern“ abzubauen. $\mathrm{Zu}$ mindest in dieser Hinsicht sind uns die USA weit voraus, da dort seit jeher der „fresh start" und nicht der mittelalterliche Schuldturm im Mittelpunkt der Betrachtung steht.

$\mathrm{Da}$ angelsächsische Interessensvertreter die Gunst der Stunde nutzen möchten, um das deutsche Insolvenzrecht so grundlegend zu ändern, dass am Ende ein von Großgläubigerpartikularinteressen bestimmtes Verfahren übrig bleibt, in dem unabhängige Gerichte und Insolvenzverwalter ersetzt sind, ist besondere Aufmerksamkeit geboten. Denn die Tatsache, dass der seit dem römischen Recht geltende Grundsatz der Gläubigergleichbehandlung und damit ein zentraler Teil unserer Insolvenzrechtskultur verloren gehen würde, wird von Interessensgruppen bewusst in Kauf genommen.

\section{Rechtliche und empirische Grundlagen}

Eine Sanierung in der Insolvenz geht - auch wenn es in der Öffentlichkeit, gerade wenn Arbeitnehmerinteressen betroffen sind, oft verdrängt wird - nur unter Beachtung der gesetzlichen Ziele des Insolvenzverfahrens. Diese sind in $\mathbb{} 1$ InsO festgelegt, wonach das Insolvenzverfahren dazu dient, die Gläubiger eines Schuldners gemeinschaftlich zu befriedigen, indem das Vermögen des Schuldners verwertet und der Erlös verteilt oder in einem Insolvenzplan eine

4 So gaben dies der Maschinenbauer Heidelberger Druck (http://www.teleboerse.de/ nachrichten/-HeidelbergDruck-noch-rot-article718436.html), MAN Nutzfahrzeuge (http://www.n-tv.de/wirtschaft/Umsatzeinbruch-fuer-MAN-article441038.html), Würth (http://www.topnews.de/-schwierige-situatiion-der-kunden-bringt-schraubenkonzern-wuerth-erheblichen-umsatzeinbruch-ein-369249) bekannt. 
abweichende Regelung insbesondere zum Erhalt des Unternehmens getroffen wird. Es handelt sich um ein kollektives Gesamtvollstreckungsverfahren im Interesse der Gläubiger. Die Sanierung des Schuldners ist ein Reflex, wenn es den Gläubigern dient, nicht aber der Hauptzweck des Verfahrens. Es ist das auch betriebswirtschaftliche - Verständnis, dass ein Unternehmen nur saniert werden soll, wenn der Fortführungswert höher ist als der Zerschlagungswert. ${ }^{5}$ Denn Unternehmenssanierung ist kein Selbstzweck, sondern ein Mittel der Gläubigerbefriedigung. ${ }^{6}$ Der Charakter als Gesamtvollstreckungsverfahren führt dann zu einem Zielkonflikt, wenn Investoren den Erhalt von Arbeitsplätzen postulieren, aber gleichzeitig nicht einmal gewillt sind, die Zerschlagungswerte zu bezahlen. Demgemäß gibt es zunehmend Stimmen, die den Arbeitnehmerinteressen eine größere Bedeutung im Rahmen der Entscheidungsfindung über den ökonomischen Ausgang eines Insolvenzverfahrens zuweisen wollen ${ }^{7}$, da der Insolvenzverwalter - de lege lata - unter dem Zerschlagungswert nicht verkaufen kann. ${ }^{8}$

Die Ausrichtung als kollektives Vollstreckungsverfahren steht im Gegensatz zum amerikanischen Insolvenzrecht, wo es nicht um den Schutz der Gläubiger, wie in Deutschland, sondern vor allem um den Schutz vor den Gläubigern und damit einen „fresh start“ für den Schuldner geht. Aber auch in den USA hat man zwischenzeitlich erkannt, dass Missbrauchsmöglichkeiten in der Vergangenheit Tür und Tor geöffnet waren. Deshalb wurde im Jahre 2005 der Bankruptcy Abuse Prevention and Consumer Protection Act (BAPCPA) eingeführt, um u. a. die Verweildauer von Unternehmen unter dem Schutz des Insolvenzrechts zu verkürzen und vor allem den Gläubigern, die die Schäden letztlich zahlen müssen, mehr Rechte einzuräumen. ${ }^{9}$

Die einzige Möglichkeit der Eigensanierung im deutschen Recht aus Sicht des Gesellschafters eines insolventen Unternehmens ist der Insolvenzplan. Dessen Problem ist, dass derzeit ein Eingriff in die Gesellschafterrechte mittels Insolvenzplan nicht möglich ist und das Insolvenzplanverfahren nur innerhalb des einheitlichen und bereits eröffneten Insolvenzverfahrens initiiert werden kann. Anreize mit Hilfe eines „prepacked plan“ unter Nutzung von $\$ 18 \mathrm{InsO}$ frühzeitig Insolvenzantrag zu stellen, klingen gut, bieten aber reichlich Raum für negative Überraschungen. Denn für den schuldnerischen Planersteller gibt es - im Gegensatz zum amerikanischen Recht - keine Exklusivperiode, in der nur er sich um die Sanierung seines Unternehmens mittels Reorganisationsverfahren bemühen kann. In Deutschland ist es an der Tagesordnung, dass sich

5 So auch Wellensiek/Schluck-Amend, GmbH in Krise, Sanierung und Insolvenz, Rdn. 4.13.

6 Ebenda.

7 http://www.bild.de/BILD/regional/duesseldorf/aktuell/2009/10/28/karstadt/wievielmillionen-streicht-der-insolvenzverwalter-ein.html.

8 Siehe auch Wellensiek/Schluck-Amend, aaO (Fn. 5), Rdn. 4.13.

9 U.S. Department of Justice, BAPCPA: www.justice.gov/ust/eo/bapcpa/index.htm. 
ein Insolvenzverfahren völlig anders entwickelt als vom Schuldner und dessen Beratern erwartet. Der Schuldner hat keinerlei Kontrolle über den Prozess und wird von den Geschehnissen meist völlig überrollt. Bestellt das Gericht beispielsweise einen Verwalter, der auch 11 Jahre nach dem Inkrafttreten der InsO noch keinen Insolvenzplan erstellt hat, so ist gewiss, dass jeder auch noch so gut vorbereitete Insolvenzplan keine Realisierungschance haben wird, da sich immer Gründe finden lassen, warum ein Planverfahren im konkreten Falle unpassend ist.

Oft unterschätzen Schuldner auch völlig, dass mit der Insolvenzantragstellung ein Zeitenwechsel eintritt. Die Zusammenarbeit mit den bisherigen Kreditsachbearbeitern findet ein abruptes Ende, da die Abwicklungsspezialisten der Banken die Geschäftsbeziehung nur mehr aus dem Blickwinkel der bestmöglichen Sicherheitenrealisierung sehen. Der erhoffte Plan ist nicht selten bereits einige Tage nach dem Insolvenzantrag Makulatur.

Um sich Verbesserungsmöglichkeiten im deutschen Insolvenzrecht zu nähern, ist es wichtig, sich mit den empirischen Fakten auseinanderzusetzen, da von interessierter Seite oft der falsche Eindruck erweckt wird, als wäre jeder Betrieb zu retten, wenn die Beteiligten dies nur wollten. ${ }^{10}$

Da sich auch die Diskussionsbeiträge angelsächsischer Interessenvertreter fast ausschließlich auf die wenigen medienträchtigen Großverfahren beziehen, wird deutlich, dass Prof. Hirte's These $2^{11}$ unbeachtet lässt, dass sich mehr als $99 \%$ aller bundesdeutschen Insolvenzverfahren in Unternehmen abspielen, die einen Umsatz von weniger als $€ 50$ Mio. pro Jahr aufweisen. Nachweislich hatten im Jahre 2003, dem Scheitelpunkt der letzten Insolvenzwelle, von 39.700 Insolvenzverfahren nur $0,3 \%$ der betroffenen Unternehmen einen Umsatz von mehr als € 50 Mio. ${ }^{12}$. Dies bedeutet, dass bei allen Änderungswünschen nicht vergessen werden darf, dass es unzählige Marktaustrittsverfahren und Kriminalverfahren gibt, die aus Sicht von internationalen Großkanzleien und Investmentbanken zwar wirtschaftlich völlig uninteressant sind, jedoch dennoch geordnet abgewickelt werden müssen. Eine Abwicklung durch die bisherigen Organe ist in solchen Fällen undenkbar.

10 Z.B. http://www.welt.de/wams_print/article4015031/In-Essen-organisieren-sich-dieGlaeubiger.html.

11 In der Insolvenz einer Kapitalgesellschaft bedarf es grundsätzlich weder eines gerichtlichen Beschlusses über die Eröffnung eines Insolvenzverfahrens noch ist die Einsetzung eines Fremdverwalters erforderlich. Ausreichend ist, dass sich mit Eintritt der materiellen Insolvenz die Pflichtenbindung der Gesellschaftsorgane ändert: Sie „dienen“ von diesem Zeitpunkt an nicht mehr den Gesellschaftern, sondern den Gläubigern, wie sich das jetzt schon aus $₫ 64$ (früher Abs. 2) GmbHG und $₫ 93$ Abs. 3 Nr. 6 AktG i. V. m. \} 9 2 \text { Abs. } 2 \text { (früher Abs. 3) AktG ergibt. }

12 Vgl. Creditreform 2003, S. 11. 
Da die Insolvenz zum marktwirtschaftlichen Wirtschaftsleben so untrennbar gehört, wie der Tod zum Leben, ist der Verfasser im Gegensatz zu Prof. Hirte's These $1^{13}$ der Auffassung, dass die Insolvenz immer zeitgemäß ist. Denn solange es Kredite und marktwirtschaftliche Selbstregulierung gibt, gibt es Insolvenzen. Auch der von Prof. Hirte vorgeschlagene Verzicht auf ein gerichtlich organisiertes Insolvenzverfahren bei Fehlen eines Fremdantrages ist nicht möglich. Im Gegensatz zur Gesellschaftsgründung, die zumindest bei den juristischen Personen nicht ohne Publizität einhergeht ${ }^{14}$, kann die Insolvenz nicht lautlos und in einem Geheimverfahren vonstatten gehen. Der Rechtsverkehr muss wissen, ob er mit einem insolventen Vertragspartner kontrahiert, damit der jeweilige Marktteilnehmer selbstbestimmt entscheiden kann, welche Risiken er eingehen will. Denn dass es erhebliche Risiken im Geschäftsverkehr mit insolventen Unternehmen gibt, hat der Fall Quelle gerade wieder eindrucksvoll verdeutlicht. ${ }^{15}$

\section{USA als Vorbild?}

Im Rahmen der Finanz- und Wirtschaftskrise sind die Möglichkeiten, die ein nationales Insolvenzrecht für die Sanierung und den Erhalt von Arbeitsplätzen bereitstellt, von besonderem Interesse; hierbei wird oft das US-amerikanische Insolvenzrecht als Vorbild genannt. Das US-amerikanische Insolvenzrecht ist im sogenannten Bankruptcy Code (BC), dem 11. Titel des United States Code (USC) kodifiziert. Die erste gesetzliche Regelung erfolgte durch den Bankruptcy Act von 1898, heute ist der Bankruptcy Code von 1978 für das amerikanische Insolvenzgeschehen maßgeblich.

Die Ursprünge des Reorganisationsverfahrens gehen zurück auf den Zusammenbruch von Eisenbahngesellschaften Ende des 19. Jahrhunderts, deren Anlagevermögen vor allem aus Zügen und Schienen bestand, die einen nur unverhältnismäßig geringen Liquidationswert gehabt hätten, weshalb richterrechtlich das receivership-Verfahren entwickelt wurde. ${ }^{16}$

Einer der primären Zwecke des Bankruptcy Act ist es, den redlichen Schuldner von seiner erdrückenden Schuldenlast zu befreien und ihm einen „fresh start“ $\mathrm{zu}$ ermöglichen. ${ }^{17} \mathrm{Da}$ in den USA die Sozialsicherungssysteme kaum vor-

13 Der insolvenzmäßigen Abwicklung von Gesellschaften liegt ein regulatorischer Ansatz zugrunde, der in anderen Bereichen des Gesellschaftsrechts (so) nicht mehr als zeitgemäß angesehen wird.

14 Vgl. z.B. $\$ 7$ Abs. 1 GmbHG; $\$ 36$ Abs. 1 AktG.

15 Vgl.http://www.faz.net/s/RubD16E1F55D21144C4AE3F9DDF52B6E1D9/ Doc EAC7 A8 A6DE2854C67B2C55ED5B6D53D81 ATpl Ecommon Scontent.html.

16 Braun, in: Nerlich/Römermann, Komm. z. InsO, Vorb. $\$ \$ 217-169$, Rdn. $36 \mathrm{ff}$.

17 Römermann, in: Nerlich/Römermann, Komm. z. InsO, Vorb. $\int \mathbb{S} 286-303$, Rdn. $37 \mathrm{ff}$. 
handen sind, soll mit Hilfe des Bankruptcy Act auch ein völliger sozialer Absturz verhindert werden. Zudem soll die unternehmerische Risikobereitschaft ausdrücklich belohnt werden, indem die realistische Chance auf einen Neubeginn in Aussicht gestellt wird. ${ }^{18}$ Das US-amerikanische Insolvenzrecht kennt je nach Ziel des Insolvenzverfahrens zwei grundsätzlich verschiedene Weichenstellungen: Die Abwicklung bzw. Liquidation eines Unternehmens gemäß Chapter 7 sowie die Reorganisation bzw. Neuaufstellung eines Unternehmens gemäß Chapter $11 .{ }^{19}$

In Deutschland geht das Insolvenzgeschehen der Neuzeit zurück auf die Konkursordnung von 1877. Die Konkursordnung war eines der vier Reichsjustizgesetze, die das Deutsche Reich erst zum einheitlichen Rechtspflegegebiet machten. Sie war mehr als $120 \mathrm{Jahre}-$ mit nur geringen Änderungen - in Kraft, was umso erstaunlicher ist, wenn man sich vergegenwärtigt, dass dies einen Zeitraum vom Beginn der Industrialisierung bis ins Internetzeitalter abgebildet hat. Auch heute, 11 Jahre nach Inkrafttreten der InsO, ist die Insolvenzordnung meist keine Basis für einen „fresh start“, sondern führt häufig zum wirtschaftlichen und gesellschaftlichen Untergang des Schuldners. So wünschenswert es auch wäre, die Insolvenz als echte Chance begreifen zu können, so nüchtern ist aktuell die gesellschaftliche Akzeptanz zu sehen. ${ }^{20}$

Um Verbesserungsmöglichkeiten im deutschen Recht zu erkennen, ist es aufschlussreich, die Anzahl der Insolvenzgerichte in beiden Staaten zu vergleichen, da die Diskussion um eine Zuständigkeitskonzentration der bundesdeutschen Insolvenzgerichte und die fehlende Spezialisierung gerade kleiner Insolvenzgerichte einen der zentralen Kritikpunkte am deutschen Insolvenzrecht darstellt. In den USA gibt es lediglich 94 Insolvenzgerichte, jeder District Court ist ausschließlich für alle Insolvenzsachen eines Districts zuständig. In Deutschland gibt es 182 Insolvenzgerichte, alleine in Bayern sind es 29. Setzt man die jeweilige Anzahl der Insolvenzgerichte in den beiden Staaten ins Verhältnis zu einzelnen Kennziffern, dann wird der Unterschied an Spezialisierung greifbar.

Die USA hat eine Fläche von 9,8 Mio. km², ein BIP in 2008 von 14.265 Mrd. US\$, 308 Mio. Einwohner und lediglich 94 Insolvenzgerichte, was bedeutet, dass ein Insolvenzgericht pro $104.255 \mathrm{~km}^{2}$ und 3,3 Mio. Einwohner zuständig ist. Die Spezialisierung wird durch die dauerhafte Amtsausübung amerikanischer Insolvenzrichter noch verstärkt. So wurde der bekannte und auch international außerordentlich geschätzte Bankruptcy Judge, Hon. Arthur J. Gonzalez, mit Court Order des United States Court of Appeals für den

18 Ebenda.

19 Grauke/Youdelman, Münchener Komm. z. InsO, Anhang, Länderberichte, Vereinigte Staaten von Amerika, Rdn. $6 \mathrm{f}$.

20 http://www.focus.de/finanzen/news/quelle-aus-landesregierung-greift-insolvenzverwalter-an_aid_446730.html. 
Zeitraum vom 9. Oktober 2009 bis 10. Oktober 2023 für den Southern District of New York bestätigt. ${ }^{21}$

In Deutschland kommen auf eine Fläche von 0,36 Mio. km², ein BIP in 2008 von $2.491 \mathrm{Mrd}$. $€$ (3,641 Mrd. US\$), 82 Mio. Einwohner, 182 Insolvenzgerichte, was bedeutet, dass ein Insolvenzgericht pro $1.978 \mathrm{~km}^{2}$ und 450.500 Einwohner zuständig ist. Diese Zersplitterung des bundesdeutschen Insolvenzgeschehens, wo de facto alle $60 \mathrm{~km}$ ein unterschiedliches Insolvenzregime gilt, stellt eines der Probleme des deutschen Insolvenzgeschehens dar. Ferner müssen interessierte Richter die Möglichkeit erhalten, bei angemessener Besoldung über einen längeren Zeitraum am Insolvenzgericht zu verbleiben und dort den Karriereweg zu bestreiten.

\section{Ist die Überschuldung als Insolvenzgrund noch zeitgemäß?}

In Deutschland ist nicht nur ein Insolvenzantrag sondern auch ein Eröffnungsgrund erforderlich. Insolvenzgründe sind drohende Zahlungsunfähigkeit gemäß $\mathbb{1} 18$ InsO, Zahlungsunfähigkeit gemäß $\ 17$ InsO sowie Überschuldung gemäß $\ 19$ InsO, wobei von der Möglichkeit bei drohender Zahlungsunfähigkeit Insolvenzantrag zu stellen, wegen des Stigmas der Insolvenz und der fehlenden Vorhersehbarkeit der Verwalterbestellung, kein Gebrauch gemacht wird. Berechtigterweise stellt Herr Prof. Hirte in seiner These $4^{22}$ daher die Sinnhaftigkeit des Insolvenzgrundes der Überschuldung in Frage.

Nach Auffassung des Verfassers hängt die Beantwortung dieser Frage davon $\mathrm{ab}$, welche rechtspolitische Überzeugung man als vorzugswürdig erachtet. Soll ein überschuldetes Unternehmen bis zum letzten Cent in der bloßen Hoffnung auf Besserung weiterwirtschaften können oder soll das Restvermögen ab dem Eintritt der Überschuldung für die Gläubiger gesichert und weitere Schäden - vor allem auch für Neugläubiger - vermieden werden?

Aus Sicht der Insolvenzpraxis ist der Insolvenzgrund der Überschuldung nicht entscheidend, da deswegen in der Vergangenheit fast nie ein Antrag gestellt wurde. ${ }^{23}$ Man sollte nur ehrlich genug sein, um zuzugeben, dass die aus dem angelsächsischen Rechtskreis angepriesene nachlaufende Haftung der Directors für fraudulent oder wrongful trading ${ }^{24}$ keine Lösung im inner-

21 Order of the United States Court of Appeals for the Second Circuit, 9. Juli 2009.

22 Die entscheidende Steuerungswirkung hat ein auf den Insolvenzgrund der Zahlungsunfähigkeit beschränktes Fremdantragsrecht.

23 So wurden nach Auskunft des Statistischen Bundesamts beispielsweise im Jahr 2007 bei 29.160 Unternehmensinsolvenzverfahren lediglich 630 Eröffnungsanträge (2,2 \%) wegen Überschuldung gestellt.

24 Vgl. Schlegel, Münchener Komm. z. InsO, Anhang Länderberichte: England und Wales, Rdn. 38 . 
deutschen Recht ist. Zum einen funktioniert dies bereits in angelsächsischen Ländern nicht, zum andern hilft es niemandem weiter, Geschäftsführer wegen neuer Ansprüche nach Insolvenzreife kostspielig in Regress zu nehmen, wenn diese wegen vorinsolvenzlicher Haftungstatbestände bereits vermögenslos sind.

Es darf aber nicht unterschätzt werden, dass die Strafbarkeit der Insolvenzverschleppung zumindest einige Fremdorgane davon abhält, alles zu tun, was Kapitaleigner von ihnen fordern. Ohne den Insolvenzgrund der Überschuldung laufen Gläubiger Gefahr zusätzliche Schäden zu erleiden.

\section{Verfabrenskontrolle als Sanierungshebel?}

Das US-amerikanische Insolvenzverfahren ist einzuleiten mit einem Eröffnungsantrag, welcher sowohl vom Schuldner (voluntary petition) als auch von einem oder mehreren Gläubigern (involuntary petition) gestellt werden kann. Im Unterschied zum deutschen Insolvenzverfahren führt der Insolvenzantrag direkt zu einer formellen Eröffnung des Verfahrens ohne weitere gerichtliche Prüfung, wenn der Schuldner geeignet ist, das Insolvenzverfahren durchzuführen und weitere formelle Anforderungen erfüllt sind, sog. order for relief. Der Fremdantrag muss von mindestens drei Gläubigern gestellt werden, deren Forderungen insgesamt US\$10.000,- übersteigen ${ }^{25}$, wobei der Rechtsschutz nicht automatisch eintritt, sondern die order for relief der ausdrücklichen gerichtlichen Anordnung bedarf. Widerspricht der Schuldner der Eröffnung des Verfahrens ist ein Gerichtsverfahren und die Feststellung eines Eröffnungsgrundes erforderlich. Im Gegensatz zum deutschen Recht bestehen keine gesetzlichen Insolvenzantragspflichten, es gibt jedoch den Grundsatz der Treuepflicht gegenüber Gesellschaftern und Gläubigern („fiduciary duty"). ${ }^{26}$ Der BGH hat im Rahmen der Schnellverschlusskappenentscheidung ganz aktuell und ummissverständlich entschieden, dass das Chapter 11 Verfahren als Eröffnung eines ausländischen Insolvenzverfahrens anerkannt wird, obwohl die prägenden Strukturelemente der beiden Insolvenzordnungen gravierende Unterschiede aufweisen. ${ }^{27}$

Interessant ist, ob sich aus der Verfahrenskontrolle, die in den USA und Deutschland diametral unterschiedlich geregelt ist, Verbesserungen für den deutschen Insolvenzstandort Deutschland ergeben können. Bei Unternehmenssanierungen in den USA ist das verwalterlose Verfahren die Regel, der Schuldner handelt als debtor in possession und ist zur Erledigung der täglichen

25 Vgl. dazu grundlegend Weintraub/Resnick, Involuntary petitions under the new Bankruptcy Code, Banking Law Journal, Volume 97, 1980, $292 \mathrm{ff}$.

26 Vgl. Bull, ZIP 1980, 843, 845; Meyer-Löwy/Poertzgen/Eckhoff, ZInsO 2005, 735, 736.

27 BGH Urt. vom 13. Oktober 2009 - X ZR 79/06. 
Geschäfte befugt, während die Kontrolle durch Gläubigerausschüsse erfolgt. ${ }^{28}$ $\mathrm{Da}$ der Schuldner in den USA einen Insolvenzantrag ohne Vorliegen eines Insolvenzgrundes stellen kann, um sich damit zur Wahrung eigener Interessen unter den Schutz des Insolvenzrechts zu flüchten, ist dies auch verständlich. In Deutschland erfolgt die Durchführung des Insolvenzverfahrens mit einem Insolvenzverwalter und zwar nur, wenn ein gesetzlicher Insolvenzgrund vorliegt. Die Frage der Verfahrenskontrolle setzt somit an ganz anderen Zeitpunkten der unternehmerischen Fehlentwicklung ein, was meist übersehen wird, wenn die Eigenverwaltung im inländischen Recht als gewünschter Regelfall propagiert wird. ${ }^{29}$

Die Eigenverwaltung durch den Schuldner ist zwar rechtlich möglich, jedoch nicht ohne gerichtlich bestellte Aufsichtsperson, den Sachwalter. Dies ist verständlich, da das Unternehmen - im Gegensatz zu den USA - auch wirtschaftlich insolvent ist. In der Praxis hat sich herausgebildet, dass ein Antrag auf Eigenverwaltung in kleinen Verfahren meist aussichtslos ist und in Großverfahren nur dann Aussicht auf Erfolg hat, wenn eine anerkannte Sanierungspersönlichkeit in die Geschäftsführung berufen wird. ${ }^{30}$

In diesem Kontext ist es interessant, Prof. Hirte's These 2 zu beleuchten, d. h. ob es wirklich genügen kann, lediglich die materiell-rechtliche Pflichtenbindung des Geschäftsführers zu ändern. Eine an sich verlockende Vorstellung, da minimal-invasiv und scheinbar kostengünstig. Leider geht diese Auffassung an der Realität der Insolvenzpraxis völlig vorbei. Geschäftsführer, vor allem Gesellschafter-Geschäftsführer, die nicht selten einschließlich ihrer Familienmitglieder für das insolvente Unternehmen aufgrund von Personalsicherheiten einstehen müssen, können nicht einfach einen Hebel im Kopf umlegen, um fortan nur mehr die Interessen der Gläubiger zu vertreten. Im Gegenteil: In der Praxis setzen diese Personen - menschlich nachvollziehbar - alles daran, nicht völlig unterzugehen.

Bereits im römischen Recht wurde daher erkannt, dass sich die jedem Konkurs latent innewohnende Anarchie nur lösen lässt, wenn ein unabhängiger Dritter mit der Vermögensverwertung betraut wird. ${ }^{31}$ Das lateinische Wort Konkurs (concurrere=zusammenlaufen) verdeutlicht die Problemlage fast schon kör-

28 Vgl. Moller/Foltz, Chapter 11 of the 1978 Bankruptcy Code, North Carolina, Law Review, Volume 58, 1980, $881 \mathrm{ff}$.

29 Vgl. Vierbuchen, Handelsblatt vom 5. Juni 2003, S. 16; Spies, ZInsO 2005, 1256; VALLENDER, in: Karsten Schmidt/Uhlenbruck, Die GmbH in Krise, Sanierung u. Insolvenz, 2003, S. 822; Uhlenbruck, DZWIR 2000, 18; Buchalik, NZI 2000, 295; Zybon, BFuP (Betriebswirtschaftliche Forschung und Praxis) 1986, 419. Vgl. auch Westrick, NZI 2003, 65; WitTig, Münchener Komm. z. InsO, 2003, Vorb. $\int \$ 270$ bis 285 Rdn. 7 f.

30 Vgl. Bales, NZI 2008, 216; http://www.wiwo.de/unternehmen-maerkte/ihr-platz-insolvenz-in-eigenverwaltung-109613/; http://www.indat-report.de/titel/titel_06_01.htm.

31 Braun/Kießner, Komm. z. InsO, 3. Aufl., 2007, Einführung, Rdn. 4. 
perlich spürbar, denn alle Prätendenten wollen das Wenige, das noch übrig ist, und zwar nur für sich alleine.

Aber auch Fremdgeschäftsführer sind oft nur sehr bedingt geeignet in der Insolvenz weiterzuarbeiten, denn ohne eine klare Perspektive, die in der Insolvenz nur sehr selten anzutreffen ist, will dieser Personenkreis meist nur schnell weg, um die eigene Vita nicht mehr mit einem insolventen Unternehmen zu belasten. ${ }^{32}$ Ein eindrucksvolles Beispiel für diese These ist u.a. das international bekannte US-Insolvenzverfahren Kmart Corp., bei welchem 22 Executives im Dezember 2001 „Retention Loans“ in Höhe von US\$28,5 Mio. gewährt wurden. Nachdem im Januar 2002 das vorbereitete Chapter 11 Verfahren initiiert worden ist und es der erklärte Wunsch war, dass die Executives im Unternehmen verbleiben, verließen trotzdem 16 von 22 Executives das Unternehmen bis Ende März 2002, was zu gerichtlichen Maßnahmen der Gläubiger und viel Ärger führte. ${ }^{33}$

\section{Einzelprobleme}

Ein zentrales und hinlänglich diskutiertes Problem der deutschen Insolvenzpraxis ist, dass ein Insolvenzplanverfahren auf Grundlage der aktuellen Gesetzeslage nur als Konsensverfahren möglich ist. Dies gilt sowohl gegenüber den Altgesellschaftern wie auch den Gläubigern und ist gleichermaßen unerfreulich. Diese Tatsache ist die Konsequenz der fehlenden Zugriffsmöglichkeit auf die wertlosen Gesellschaftsanteile. Durch den fehlenden Zugriff auf die Geschäftsanteile wurde den Altgesellschaftern, die gerade nicht bereit oder in der Lage waren, die Insolvenz durch Nachschießen von Geldmitteln zu verhindern, der Ausgang des Planverfahrens de facto in die Hand gelegt. Ein unbefriedigendes Ergebnis, welches ein Grund für den bisherigen sehr begrenzten Erfolg des Insolvenzplanverfahrens und des „forum shoppings“ nach England ist. Letzteres hat sich momentan jedoch erledigt, da seit dem $\mathrm{Zu}$ sammenbruch von Lehman Brothers kein Geld mehr für solche Gestaltungen vorhanden ist. Da den Gläubigern ohne Not und vor allem ohne echten Grund der Mehrwert einer going-concern Sanierung genommen wurde, ist es nach 11 Jahren Insolvenzordnung an der Zeit den Debt-to-Equity-Swap im gerichtlichen Verfahren endlich zuzulassen. ${ }^{34}$

Ein weiteres Sanierungshemmnis im Planverfahren ist die ausgeprägte Rechtsmittellastigkeit. Die Erfahrungen der Restrukturierungspraxis haben gezeigt, dass aufgrund von Rechtsmittelverfahren kaum absehbare Zeitver-

32 Vgl. Paulus, DStR 2002, 1865.

33 Vgl. BusinessWeek, 4. Juli 2005, http://www.businessweek.com/magazine/content/05_27/b3941102_mz020.htm.

34 Vgl. Jaffé/Friedrich, ZIP 2008, 1854; Westpfahl/Janjuah, Beilage zur ZIP 3/2008, 15. 
zögerungen möglich sind. Störer haben es leicht, da das Insolvenzplanverfahren sehr formalisiert ist und fast alle wichtigen Vorschriften als zwingendes Recht ausgestaltet sind. Selbst kleine Unaufmerksamkeiten können einem obstruierenden Gläubiger die Aussicht auf ein langjähriges Rechtsmittelverfahren eröffnen. Der Konflikt liegt hierbei zwischen den legitimen Interessen der Beschwerdeberechtigten an der Wahrung ihrer Rechte und den schwerwiegenden Folgen aufgrund einer Zeitverzögerung durch die Einlegung von Rechtsmitteln für die Sanierung des Unternehmens. Da aber die übrigen Beteiligten ein legitimes Interesse an einer zügigen Umsetzung des bestätigten Insolvenzplans und damit der zeitnahen Auszahlung der Planquote haben, muss der Rechtsweg beschränkt oder außerhalb des Insolvenzverfahrens verlagert werden. ${ }^{35}$

Zum Abbau der Rechtsmittellastigkeit des Insolvenzplanverfahrens böte sich die Einführung eines Spruchverfahrens an. Im Gesellschaftsrecht können Minderheitenaktionäre mit dem Spruchverfahren die Höhe von Ausgleichsund Abfindungszahlungen bei Strukturmaßnahmen, wie ihrem Ausschluss bei einem „squeeze-out“, vor Gericht überprüfen lassen. Gesellschaftsrechtliche Maßnahmen werden aber durch den Streit über die Höhe der Kompensation nicht verzögert. Das Spruchverfahren dient dem Zweck des Minderheitenschutzes und ist das Äquivalent für den Ausschluss bestimmter Kontrollrechte. Es soll eine Abkopplung der Entschädigungsfrage erreicht werden.

Die gleiche Grundproblematik ist auch im Planverfahren gegeben, da ein Gläubiger nicht den gesamten Plan und damit die Sanierung als Ganzes in Gefahr bringen können soll. Die Kosten möglicher Spruchverfahren müssten bei der Planaufstellung und der Finanzierung bedacht werden. Ein Problem stellt jedoch die übliche Inter-omnes-Wirkung des Spruchverfahrens dar, d.h. ein Beteiligter erstreitet zugunsten oder zulasten aller betroffenen Aktionäre eine Entscheidung. Dies hat im Aktienrecht Vorteile, ist auf das Planverfahren jedoch nicht übertragbar, denn bei der Abstimmung und der Nichteinlegung von Rechtsmitteln haben Gläubiger durchaus ganz bewusst Nachteile in Kauf genommen bzw. haben im Hinblick auf eine zukünftige Geschäftsbeziehung gerade keine Rechtmittel eingelegt. Wäre im Nachhinein allen Gläubigern eine Zusatzquote eröffnet, wäre dies ein unüberschaubares finanzielles Risiko für den Schuldner. Es muss aber darauf geachtet werden, dass die Gläubiger nicht übervorteilt werden, indem Planersteller darauf spekulieren, dass sich Gläubiger gegen unangemessene Pläne nicht zur Wehr setzen können. ${ }^{36}$

35 Vgl. Jaffé/Friedrich, ZIP 2008, 1854.

36 Vgl. Simon, Komm. z. SpruchG, Einführung, Rdn. 8; Lutter/Bezzenberger, AG 2000, 433. 


\section{Vorschlagsrecht zur Sanierung}

In den USA ist der wichtigste Teil des auf die Sanierung eines insolventen Schuldners ausgelegten Reorganisationsverfahrens der Reorganisationsplan nach $\mathbb{S} 1121 \mathrm{ff}$ BC. Dieser ist eine Vereinbarung zwischen Schuldner und seinen Gläubigern darüber, wie die bestehenden Schulden beglichen und in welcher Art und Weise das Geschäft des Schuldners weitergeführt werden soll. Das Vorschlagsrecht für einen Reorganisationsplan liegt grundsätzlich sowohl beim Schuldner als auch bei den anderen Verfahrensbeteiligten, wobei der Schuldner zu jedem Zeitpunkt ein Insolvenzverfahren mit dem Vorschlag eines Reorganisationsplanes in Gang setzen oder auch während eines bereits laufenden Insolvenzverfahrens einen Reorganisationsplan vorschlagen kann.

Die Gläubiger können aber in den USA während der ersten 120 Tage nach Erlass der „order for relief“, der sogenannten „exclusive period“, kein Planverfahren in Gang setzen. Dritte sind unter Umständen für bis zu 180 Tage gehindert, einen eigenen Vorschlag vorzulegen, $\mathbb{S} 1121$ (c) (3) BC. Einem Dritten steht ferner nur dann ein Vorschlagsrecht zu, wenn die soeben genannten Fristen abgelaufen sind und ein trustee („treuhänderischer Sanierer“) bestellt ist, $\mathbb{} 1121$ (c) BC.

Es wäre im deutschen Recht durchaus erwägenswert, im Falle einer Insolvenzantragstellung bei nachweisbar nur drohender Zahlungsunfähigkeit eine vergleichbare befristete Exklusivität einzuführen, um dem Schuldner eine Chance zu geben, sich mit seinem Gläubigern zu einigen und seine Bereitschaft wirklich frühzeitig Insolvenzantrag zu stellen, ernsthaft zu belohnen.

Der Verfasser ist wie Herr Prof. Hirte in seiner These $3^{37}$ der Auffassung, dass wir kein außergerichtliches Sanierungsverfahren benötigen, jedoch ein gläubiger-autonomes modernes Vergleichsverfahren ohne starre Quotenvorgaben wie in der überkommenden VerglO durchaus nützlich sein könnte. Damit könnten finanzwirtschaftliche Unternehmenskrisen zügig, rechtstaatlich und ohne Akkordstörer bewältigt werden. Hierbei müssten die gesetzlichen Insolvenzantragsfristen bei Einleitung dieses Vergleichsverfahrens für einen befristeten und durch richterlichen Beschluss auch nochmals verlängerbaren Zeitraum ausgesetzt werden, um einen Dominoeffekt zu vermeiden. Unterbunden bleiben muss, dass sich Großgläubiger in einem gerichtlich nicht überwachten Verfahren ein Unternehmen einverleiben können. Ein Eingriff in Eigentumsrechte, wie das Gesellschaftskapital, geht auch aus europa-

37 Wir benötigen kein besonderes Sanierungsverfahren, weil sich die „vorinsolvenzliche“ Sanierung unproblematisch in das Regel-Insolvenzrecht integrieren lässt, wenn auf die öffentlichkeitswirksame Eröffnung des Insolvenzverfahrens durch das Gericht (zunächst) verzichtet wird. 
rechtlichen Erwägungen nur in einem geordneten gerichtlichen Zwangsvollstreckungsverfahren. ${ }^{38}$

\section{Stellung des Insolvenzgerichts im modernen Insolvenzgeschehen}

Ein zentraler Streitpunkt in der Diskussion ist die Frage der Verwalterauswahl und die mögliche Einflussnahme hierauf. Die Herangehensweise der deutschen Insolvenzgerichte an dieses Thema ist höchst unterschiedlich. Wenngleich alle Insolvenzgerichte zu Recht eint, dass es mit dem Selbstverständnis des - mit sehr guten Gründen - verfassungsrechtlich mit Unabhängigkeit ausgestatteten Richters nicht kompatibel ist, nur als „rubber-stamper“ herzuhalten, so verhalten sich die Gerichte in der Praxis doch sehr unterschiedlich.

Großstadtgerichte mit langjährig in Vollzeit tätigen Insolvenzrichtern haben mit diesem heiklen Thema meist geringere Berührungsprobleme als viele kleinere regionale Insolvenzgerichte. Der Grund hierfür ist einfach auszumachen. Großstadtgerichte überblicken die Insolvenzszene meist sehr gut und wissen daher, ob eine „Empfehlung“ auf sachlichen Erwägungen des Einzelfalls beruht oder einen „Freundschaftsdienst“ darstellt. Dies bedeutet jedoch nicht, dass ein solches Gericht einer Empfehlung auch Folge leisten wird. Es besteht aber fast immer die Gewähr, dass die Gläubiger mit der richterlichen Entscheidung letztlich zufrieden sind, wenn statt dem Wunschverwalter ein Verwalter der gleichen „Gewichtsklasse“ bestellt wird.

Anders ist die Situation bei einzelnen regionalen Gerichten, die oft nur einmal in einer Dekade mit einem bedeutsamen Großinsolvenzverfahren befasst sind. Hier werden Verwaltervorschläge oft negiert und nicht selten auf Verwalter zurückgegriffen, deren Selbstvertrauen die eigenen Erfahrungen und Fähigkeiten übertrifft. Bemühungen die Gläubigerinteressen wie im Detmolder Modell frühzeitig einzubinden, sind die Ausnahme und nicht die Regel. ${ }^{39}$

Die fehlende Vorhersehbarkeit der Verwalterauswahl und die immer ausufernden Bestellungslisten führen dazu, dass die Insolvenz für viele - gerade internationale - Beteiligten immer weniger eine Handlungsoption darstellt. ${ }^{40}$ Dennoch ist der Verfasser im Gegensatz zu Prof. Hirte in dessen Thesen 6

38 Vgl. RL 77/91/EWG vom 13. Dezember 1976 (ABl EG Nr. L 26 vom 31. Januar 1977, S. $1 \mathrm{ff})$.

39 Vgl. Busch, DZWIR 2004, 353.

40 Vgl. Handelsblatt vom 9. März 2009, S. 12. 
$-8^{41}$ der Auffassung, dass die Wächterfunktion des Insolvenzgerichts völlig alternativlos ist und das Insolvenzgericht nicht geschwächt, sondern gestärkt werden muss.

Es ist nach Auffassung des Verfassers zutreffend, dass den Gläubigern das Recht eingeräumt werden sollte, bereits bei der Auswahl des vorläufigen Insolvenzverwalters Gehör zu finden, da die Entscheidungsmöglichkeit der Gläubiger im Berichtstermin viel zu spät kommt. ${ }^{42}$ Entscheiden sollte aber weiterhin nur ein unabhängiges Gericht, da nur dieses Garant für Rechtstaatlichkeit und Fairness ist und nicht einzelne Großgläubiger, die ihre wirtschaftlichen Interessen optimieren wollen. Entscheidend ist die Kompetenz der Akteure, ein starker Richter wie in den USA schadet nicht, sondern schafft ganz im Gegenteil Ordnung und Vertrauen. Könnten nur einzelne Großgläubiger den Verwalter alleine bestimmen, könnte man das Anfechtungs- und Haftungsrecht gleich mit abschaffen, da Verwalter, die von diesen Instrumenten umfangreich Gebrauch machen, mit Gewissheit vom Markt gedrängt würden, da die Großgläubiger sie nicht mehr vorschlagen würden. Die auf Panel- und Podiumsdiskussionen häufig zu vernehmende Einlassung, dass Großgläubiger Anfechtungsklagen „sportlich“ sehen würden, ist schlichtweg ein Märchen.

Da das Gebührenaufkommen der Länder im Kontext der Insolvenzverfahren sehr attraktiv ist und andere Länderjustizbereiche damit quersubventioniert werden, wäre es in Anbetracht der volkswirtschaftlichen Bedeutung der Entscheidungen der Insolvenzgerichte dringend notwendig, solche Gerichte, die bedeutsame Verfahren verantworten sollen, zu stärken und bezüglich Personal und Infrastruktur deutlich auszubauen. Viele bundesdeutsche Insolvenzrichter können und wollen in internationalen Großverfahren auf Augenhöhe mit ihren hochspezialisierten Kollegen in angelsächsischen Ländern agieren. ${ }^{43}$ Dies gilt gleichfalls für die Rechtspfleger, da auch diese enormen Einsatz und Engagement aufzeigen, wobei hier die Problematik nicht derart ausgebildet ist, da Rechtspfleger in der Regel langfristig in einem Gebiet tätig sind und nicht, wie dies bei Richtern üblich ist, häufig die Dezernate

41 - Es ist (allein) Angelegenheit der Gläubiger, über die Person eines etwaigen Insolvenzverwalters zu entscheiden. Staatliche Vorgaben für dessen Auswahl sind grundsätzlich verzichtbar.

- Kernaufgabe des Gerichts wird es sein, für etwaige Streitigkeiten den Zeitpunkt des Eintritts der materiellen Insolvenz für alle Beteiligten in einem kollektiven Verfahren verbindlich festzulegen.

- Das Gericht kann zudem Sanierungsmaßnahmen, die in der Phase der materiellen Insolvenz von einer Gläubigermehrheit beschlossen wurden, für alle Gläubiger für verbindlich erklären.

42 Nach $₫ 57$ InsO kann in der ersten Gläubigerversammlung ein anderer Insolvenzverwalter gewählt werden.

43 VAllender, Indat 09/2009, S. 46. 
wechseln. Es ist aber ein Unding, dass in Großinsolvenzverfahren einerseits Millionenbeträge an Gerichtskosten an die Landesjustizhaushalte bezahlt werden, andererseits viele Insolvenzrichter und Rechtspfleger Fachzeitschriften und Kommentare weiterhin aus eigener Tasche kaufen müssen, um überhaupt eine Basis für ihre Arbeit zu haben. Hier sind die Länder gefordert, Abhilfe zu schaffen, denn viele Streitpunkte der Insolvenzszene würden sich befrieden lassen, wenn der Insolvenzrichter - wie in den USA - mehr im Gravitationszentrum des Insolvenzgeschehens tätig wäre.

\section{Schlussbetrachtung}

Zusammenfassend lassen sich folgende Thesen aufstellen:

1. Das Insolvenzverfahren ist zeitgemäß und auch beim Fehlen eines Fremdantrags zwingend durchzuführen.

2. Eine weitreichende Konzentrierung sowie Spezialisierung der Insolvenzgerichte in Deutschland ist zwingend geboten. Viele bundesdeutsche Insolvenzrichter können und wollen in internationalen Großverfahren auf Augenhöhe mit ihren hochspezialisierten Kollegen in angelsächsischen Ländern agieren.

3. Der Insolvenzgrund der Überschuldung spielt zwar in der Insolvenzpraxis nur eine untergeordnete Rolle, sollte jedoch nicht abgeschafft werden, da ansonsten für die Gläubiger unnötige weitere Schäden drohen.

4. Die Durchführung eines verwalterlosen Insolvenzverfahrens ist in der deutschen Rechtsordnung solange nicht durchführbar und auch nicht wünschenswert, wie Insolvenzanträge viel zu spät gestellt werden.

5. Die Möglichkeit eines Debt-to-equity-swap im Insolvenzplanverfahren ist zwingend erforderlich und die Einführung überfällig.

6. Der Abbau der Rechtsmittellastigkeit des Insolvenzplanverfahrens durch Einführung eines Spruchverfahrens ist erforderlich und mit geringem Aufwand zu erreichen.

7. Die Einführung eines außergerichtlichen Sanierungsverfahrens ist nicht erforderlich, gleichwohl bedarf es eines gläubigerautonomen modernen Vergleichsverfahrens ohne starre Quotenvorgaben, um finanzwirtschaftliche Sanierung auch gegen Akkordstörer zu erleichtern.

8. Die Länder müssen die Insolvenzgerichte personell und wirtschaftlich weitaus mehr fördern, um die Stellung des Gerichts als unabhängigen Hüter des Verfahrens auszubauen und auch im internationalen Vergleich zu stärken. 